\title{
High Resolution Solid State NMR Evidence for Photochemical Formation of Poly(azulene)
}

\author{
Hugh D. Burrows ${ }^{\dagger}$ and Teresa Nunes*
}

Departamento de Química, Universidade de Coimbra, 3049 Coimbra, Portugal * ICTPOL/IST, Av. Prof. Gama Pinto, 2, 1699 Lisbon Codex, Portugal

(Received July 10, 1995)

KEY WORDS Poly(azulene) / Structure / Nuclear Magnetic Resonance /

Poly(azulene) is a conducting polymer, which results from the condensation of the azulene nucleus in the 1,3-positions, and its subsequent partial oxidation. Structures of azulene and polyazulene are shown in Figure 1. Both chemical ${ }^{1}$ and electrochemical ${ }^{2,3}$ methods have been reported for the preparation of this system, and electrical conductivity ${ }^{2,3}$ magnetic, ${ }^{4,5}$ optical, ${ }^{4}$ and electrochemical $^{5}$ properties reported. Electrical conductivities of the oxidized state of the order $0.01-1$ $\mathrm{S} \mathrm{cm}^{-1}$ have been reported. ${ }^{2,3}$ Theoretical calculations using a one-dimensional SCF-CO method $^{6}$ have provided information on the electronic structure, and suggest a polaron state, with the $\pi$ hole and spin separated on the 7 and 5 membered rings of the azulene systems, and bipolaron states, with positive charges confined to the seven membered rings. Studies of the magnetic properties suggest high spin density, even in the neutral state. ${ }^{5}$

During studies on the quenching of fluorescence of aromatic hydrocarbons by uranyl ion $\left(\mathrm{UO}_{2}{ }^{2+}\right)^{7}$ a black precipitate was observed to form in the presence of light with solutions of azulene in the presence of uranyl nitrate. Subsequent studies suggested that this amorphous product may be poly(azulene). ${ }^{8}$ However, it was not possible to obtain definite evidence for this. Because of the potential importance of this conducting polymer, the advantages that its photochemical production may provide in relation to device applications and the lack of high resolution NMR spectral data on this system, in this note we report structural characterization using high resolution solid state ${ }^{13} \mathrm{C}$ NMR spectroscopy.

\section{EXPERIMENTAL}

Azulene of the purest grade commercially available was used without further purification. The preparation of the azulene/uranyl nitrate photolysis product has been described in detail elsewhere. ${ }^{8}$ The ${ }^{13} \mathrm{C}$ spectra were obtained at $75.5 \mathrm{MHz}$ in $7 \mathrm{~mm} \mathrm{ZrO}_{2}$ rotors at $4.5 \mathrm{kHz}$ magic angle spinning rates on a Bruker MSL $300 \mathrm{P}$ spectrometer using ${ }^{1} \mathrm{H}$ cross polarization and dipolar decoupling (MAS-CP/DD technique). Typical values for constant time and $\pi / 2$ pulse width were $2 \mathrm{~ms}$ and $6 \mu \mathrm{s}$, respectively. Chemical shifts are reported in parts per million relative to tetramethylsilane.

$\uparrow$ To whom correspondence should be addressed.

\section{RESULTS AND DISCUSSION}

The high resolution ${ }^{13} \mathrm{C}$ MAS-CP/DD NMR spectrum was measured of solid azulene (Figure 2(a)), and spectral details are given in Table I. The chemical shifts are in good agreement with those observed in solution, ${ }^{9}$ and the bands are assigned on the basis of those observed in solution spectra. The spectrum was also recorded of the solid product of photolysis of uranyl nitrate and azulene (Figure 2(b)). The spectrum showed peaks in the same region as azulene, suggesting a closely related structure. However, there were three important differences. Firstly, the bands were very much broader than those in azulene. Secondly, in the spectral measurements, the magnetization recovery time for the photolysis product $(4 \mathrm{~s})$ was very much shorter than that with azulene $(60 \mathrm{~s})$. This is related in part to the shorter longitudinal ${ }^{1} \mathrm{H}$ relaxation times in the rotating frame $\left({ }^{\mathrm{H}} T_{1 \rho}\right)$ observed in the photolysis product. Thirdly, the intensity of the band at $118 \mathrm{ppm}$, attributed to the 1,3 carbon atoms, is reduced relative to the other peaks. In solution, studies of the longitudinal ${ }^{13} \mathrm{C}$ relaxation times of azulene in the

(a)

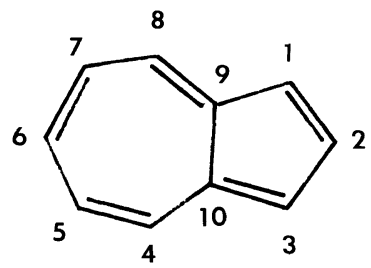

(b)

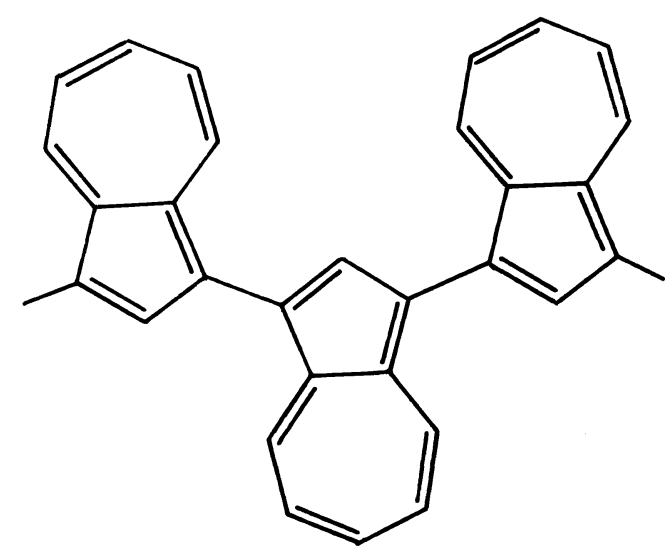

Figure 1. Structures of (a) azulene and (b) poly(azulene). 


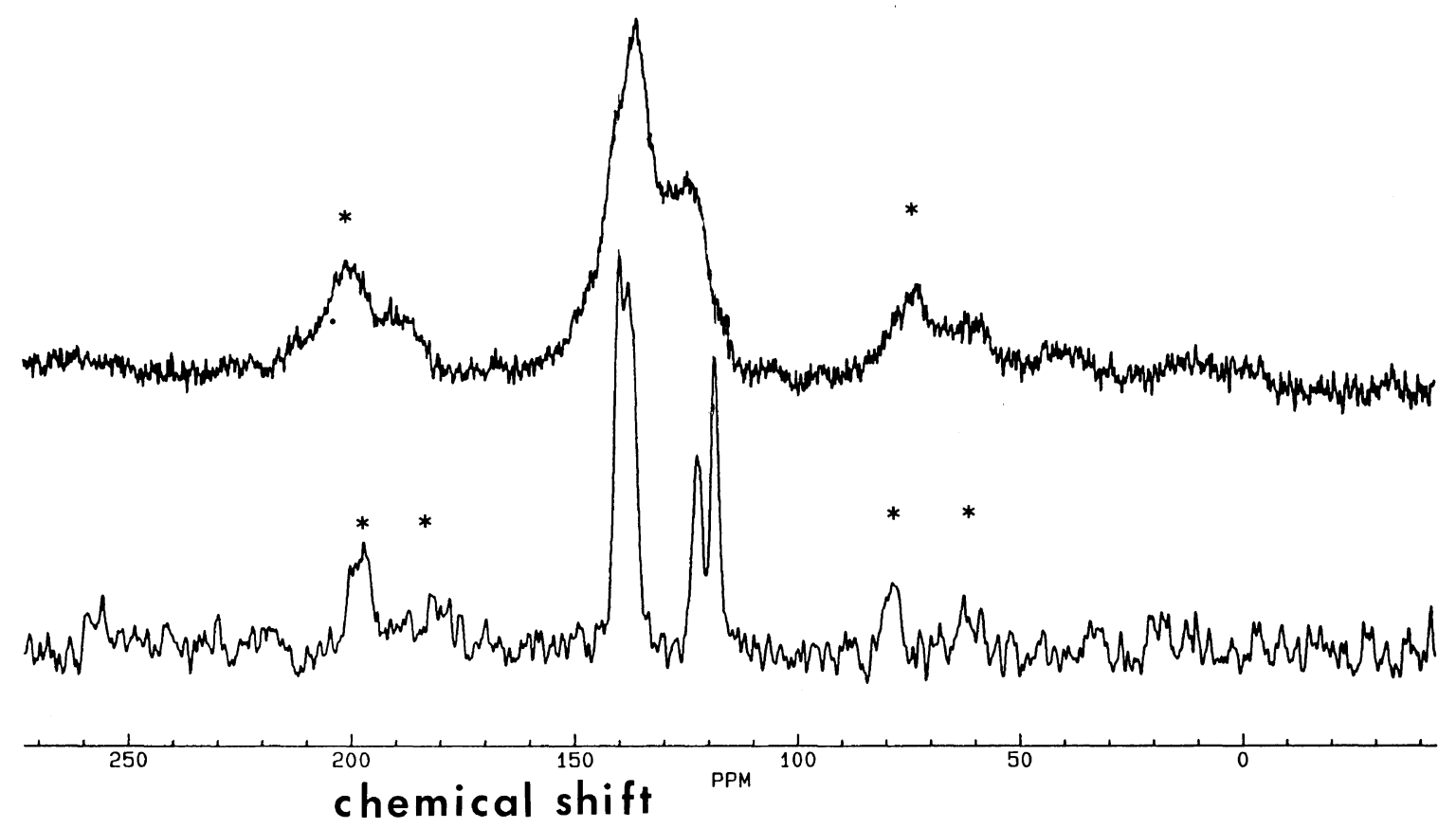

Figure 2. High resolution solid state ${ }^{13} \mathrm{C} \mathrm{CP}-\mathrm{MAS}$ spectra of (a) azulene; (b) product from photolysis of azulene in the presence of uranyl nitrate. Spinning sidebands are marked by *.

Table I. ${ }^{13} \mathrm{C}$ MAS-CP/DD spectral data for azulene in the solid state

\begin{tabular}{lcc}
\hline & \multicolumn{2}{c}{$\delta / \mathrm{ppm}^{\mathrm{a}}$} \\
\cline { 2 - 3 } Position & This work & Literature $^{\mathrm{b}}$ \\
\hline 1,3 & 118.3 & 118.9 \\
5,7 & 122.3 & 122.8 \\
$6 ; 4,8 ; 2$ & $137.5^{\mathrm{c}}$ & 137.4 \\
9,10 & 139.7 & 140.7 \\
\hline
\end{tabular}

${ }^{a}$ Relative to tetramethylsilane (TMS). ${ }^{b}$ In $\mathrm{CS}_{2}$ solution from ref 9. ${ }^{\mathrm{c}}$ Not resolved.

presence of uranyl nitrate also show the biggest difference for these carbon atoms. ${ }^{8}$ The other peaks in the five membered ring are also less intense than those in the seven membered ring. These results all indicate that the product from photolysis of azulene in the presence of uranyl nitrate is a polymer (or oligomer) derived from azulene, with bonding in the 1,3-positions, as shown in Figure 1. Addition in these positions is in agreement with the results of calculations. ${ }^{6}$ Dipolar dephasing experiments were also performed, introducing the following time delays $(\mu \mathrm{s})$ into the r.f. pulse sequence, immediately after contact: 5,20 , or 40 . The corresponding spectra are shown in Figure 3; for shorter time delays two peaks are identified, while for the longest delay only the signal obtained at the higher frequency of resonance is still observed, and thus should be assigned to all the quarternary carbons. Considering that in polynuclear aromatics the signals of nonprotonated quarternary carbons are, in general, observed at higher frequencies of resonance than the corresponding protonated ones, a polymerization reaction involving 1, 3 sites of the azulene molecule will displace the frequency of resonance of these nuclei to higher values with the consequent overlap of the resonances of all the quarternary carbons in the molecule; thus, the observed increase of the intensity of
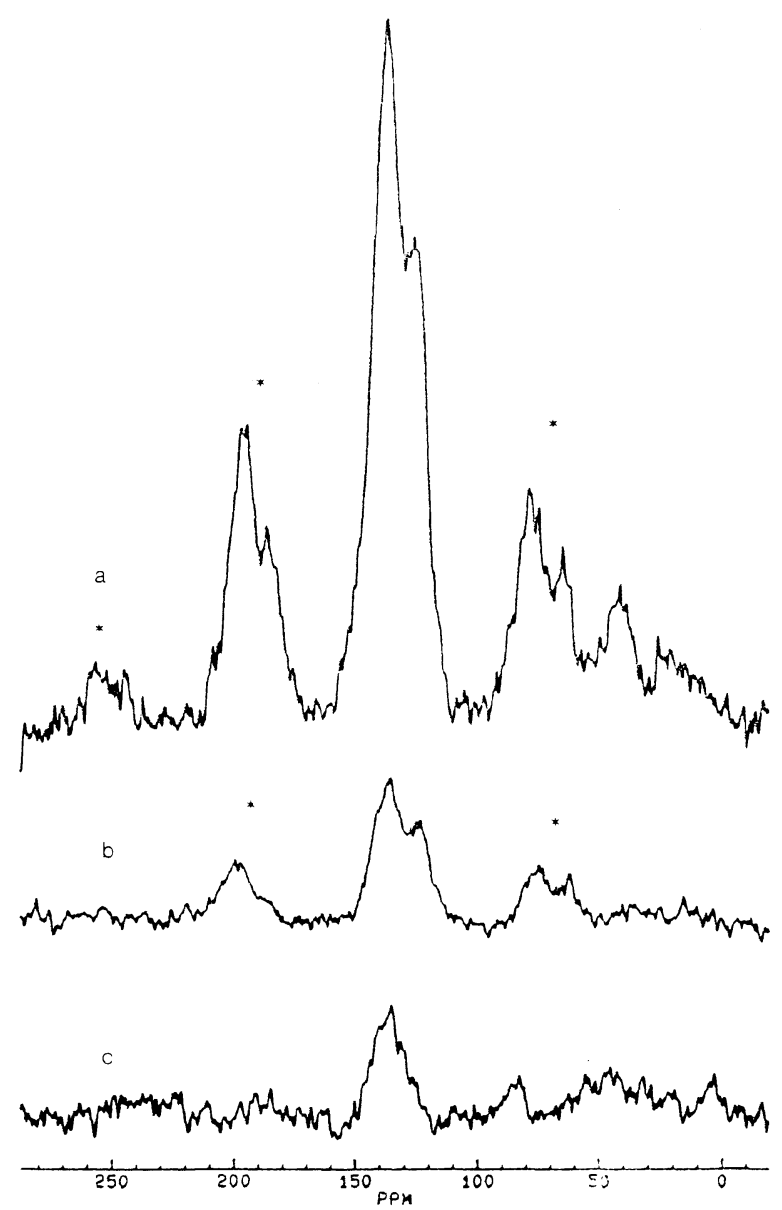

Figure 3. High resolution solid ${ }^{13} \mathrm{C}$ MAS-CP/DD spectra of the reaction product obtained with the following dipolar dephasing decays $(\mu \mathrm{s})$ : a) 5 ; b) 20 ; c) 40 . Spinning sidebands are marked by *.

the signal at higher frequency of resonance following the polymerization reaction agrees well with the structure shown in Figure 1(b). Whilst the reason for the line broadening and decrease in ${ }^{\mathrm{H}} T_{1 \rho}$ values in the 
poly(azulene) are not known, the observed product is paramagnetic ${ }^{8}$ and it is likely that a paramagnetic relaxation mechanism is strongly contributing to the decrease in the spin-spin relaxation time, that is to the line broadening; also, it is well known that shorter ${ }^{\mathrm{H}} T_{1 \rho}$ values are obtained from nuclei in less ordered structures, as is to be expected for a polymeric sample in comparison with a crystalline powder sample. In an earlier study, ${ }^{3}$ attempts were made to record the high resolution solid state ${ }^{13} \mathrm{C}$ NMR spectrum of poly(azulene). However, only a single broad peak was observed. We believe ours to be the first resolved spectrum of this product.

Recently, results have been presented for the products of photolysis of azulene in a variety of solvents. ${ }^{10}$ In chlorinated hydrocarbons, a polymer is observed, together with various other chloroazulene derivatives. It is generally believed that formation of poly(azulene) occurs via the radical cation, produced by electron transfer. Photolysis of various compounds with low ionization potentials in chlorinated hydrocarbons is known to lead to such electron-transfer, ${ }^{11}$ and we suggest that formation of this product involves a similar mechanism to that observed in the presence of uranyl nitrate, i.e., photoinduced electron transfer to produce the radical cation.
Acknowledgment. Financial support from the project ESPIRIT No. 07238 (PROTIOS) sponsored by the Commission of the European Communities is gratefully acknowledged.

\section{REFERENCES}

1. K. G. Neoh, E. T. Kang, and T. C. Tang, Polym. Bull., 19, 325 (1988).

2. G. Tourillon and F. Garnier, J. Electroanal. Chem., 135, 173 (1982).

3. J. Bargon, S. Mohmand, and R. J. Waltman, Mol. Cryst. Liq. Cryst., 93, 279 (1983).

4. S. Hayashi, S. Nakajima, K. Kaneto, and K. Yoshino, Solid State Commun., 60, 545 (1986).

5. S. Hayashi, S. Nakajima, K. Kaneto, and K. Yoshino, J. Phys. Soc. Jpn., 55, 3995 (1986).

6. K. Tanaka, M. Torjumi, S. Wanfand, and T. Yamabe, Polym. J., 22, 1001 (1990).

7. M. G. M. Miguel, S. J. Formosinho, and M. L. P. Leitão, $J$. Photochem., 33, 209 (1986)

8. H. D. Burrows, A. C. Cardoso, S. J. Formosinho, A. M. P. C. Gil, M. G. M. Miguel, B. Barata, and J. J. G. Moura, J. Photochem. Photobiol. A: Chem., 68, 279 (1992).

9. P. C. Lauterbur, J. Am. Chem. Soc., 83, 1838 (1961).

10. J. I. Selco, T. Brooks, M. Chang, M. T. Trieu, J. K. McDonald, and S. P. McManus, J. Org. Chem., 59, 429 (1994).

11. See, for example, W. C. Meyer, J. Phys. Chem., 74, 2127 (1970). 